\title{
Knowledge Capitalization with a knowledge engineering approach: the MASK method
}

Nada Matta, Jean Louis Ermine, Gérard Aubertin, Jean-Yves Trivin

“Knowledge Management and Organizational memories”, Kluwer, PP 17-28, 2002 


\title{
Knowledge Capitalization with a knowledge engineering approach: the MASK method
}

\author{
Nada Matta ${ }^{1}$, Jean Louis Ermine ${ }^{1}$, Gérard Aubertin ${ }^{2}$, Jean-Yves Trivin ${ }^{2}$
}

${ }^{1}$ Tech-CICO, Université de Technologie de Troyes

12 rue Marie Curie, BP. 2060, 10010 Troyes Cedex, France

e-mail: \{Nada.Matta, Jean-Louis.Ermine\}@utt.fr

${ }^{2}$ INRS, Institut National de recherche et de sécurité

Avenue de Bourgogne, BP. 27, 54501 Vandoeuvre Cedex, France

\{Gerard.aubertin,Jean-yves.trivin\}@inrs.fr

\begin{abstract}
Knowledge Management is a global process in the enterprise. It includes all the processes that allow capitalization, sharing and evolution of the Knowledge Capital of the firm, now recognized as a critical resource of the organization. The strategic objective of Knowledge Management is to understand, support, optimise and accelerate those processes, in coherence and crossfertilization. Knowledge engineering techniques are often used to capitalize knowledge. These techniques must be adapted and evolved considering the main objectives of knowledge management: building and use organizational memory. We study, in this paper, how the knowledge engineering method MASK (Method for Analysing and Structuring Knowledge) considers this evolution.
\end{abstract}

KEY WORDS: knowledge capitalization, knowledge engineering, MASK, knowledge sharing, modelling

\section{Introduction}

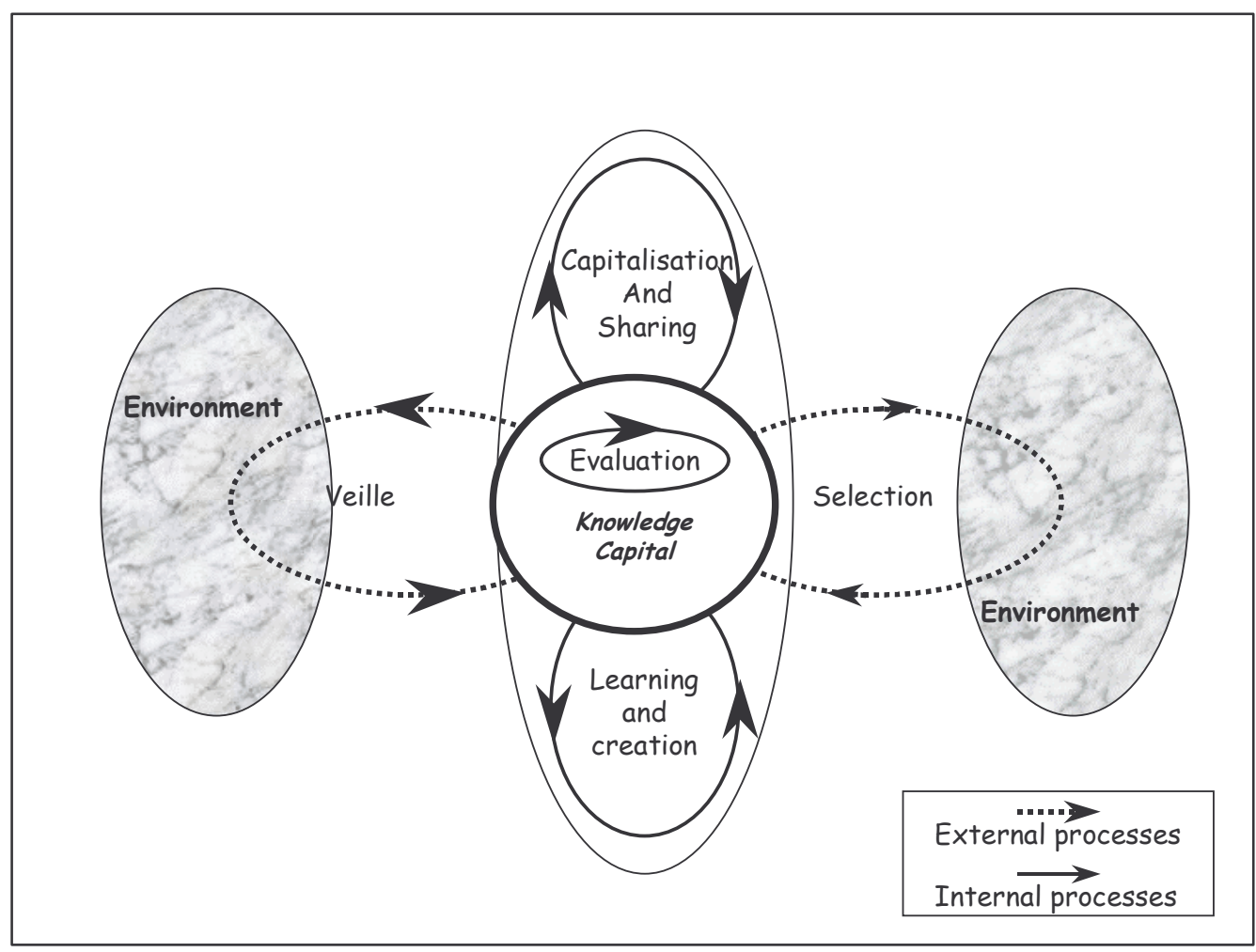

Figure 1: the key processes of KM 
Following the micro and macroeconomic evolution, the vision of the firm has drastically changed. The only useful vision was built on the production tool and the work force in the Taylor's sense. Now, important levers have appeared outside this vision: Customer Relationship, Information System, Business Intelligence, Quality, new strategic activities (sales, marketing ...). The firm is constantly changing to answer the new challenge of its competitive environment. Knowledge Management is an attempt to link the classical productive vision to these new needs. KM tries to put together, in coherent processes, the critical Knowledge which is an essential resource for the production of goods or services and the Knowledge issued from the competitive environment which is more and more important. That coherence is organised through the Knowledge Capital of the firm, where all the processes contribute, and cooperate. Those processes are organised according to the "Daisy Model" shown in Figure 1.

Those processes are internal (endogenous) as the "Capitalisation and Sharing Process" and the "Learning and Capitalisation Process". There are also external (exogenous), as Business Intelligence or Scientific or Technological Scanning which bring new Knowledge from outside, or as the Customer Relationship, or Marketing which are filters for the exponential capacity of invention and knowledge evolution within the firm.

Knowledge Management is the management of those processes, including their relationship with the Knowledge Capital of the firm. They can be described in four classes, corresponding to "petals" of the daisy and one also to the "heart" of that model.

1) The Knowledge Capitalisation and Sharing Process. It performs a "Knowledge Cycle" assuring the sharing ("recycling") of Knowledge within the company.

2) The Interaction Process, with the competitive environment. A system can't be isolated, and the Knowledge Capital is constantly fed by information from outside. The Interaction Process is the one who transforms information from outside into Knowledge useful for the company.

3) The Learning and Creation Process. This is a in the firm. It includes the problems of Learning Organisation and Creativity.

4) The Selection Process. This is an evolutionist process of selection of new ideas, new knowledge created inside the company with the help of market criteria, acceptability criteria etc. It includes the problems of marketing, customer relationship, with a strong relationship with the critical knowledge of the firm, especially the productive knowledge.

5) To those four processes, we can add a fifth, which is the Evaluation Process for evaluating the Knowledge Capital, in a qualitative or quantitative way. Methods are now designed to evaluate the Immaterial, Intellectual, or Knowledge Capital. This is still in construction.

In this paper, we focus on the Capitalisation and Sharing Process.

\section{The Capitalisation and Sharing Process}

To model that process, we refer to the well-known Nonaka and Takeuchi theory [Nonaka, 1995]. This theory considers two kinds of knowledge: Tacit Knowledge and Explicit Knowledge.

Revisiting the theory, we can easily describe the Capitalisation and Sharing Process, also called the "Knowledge Cycle", as shown in Figure 2. 


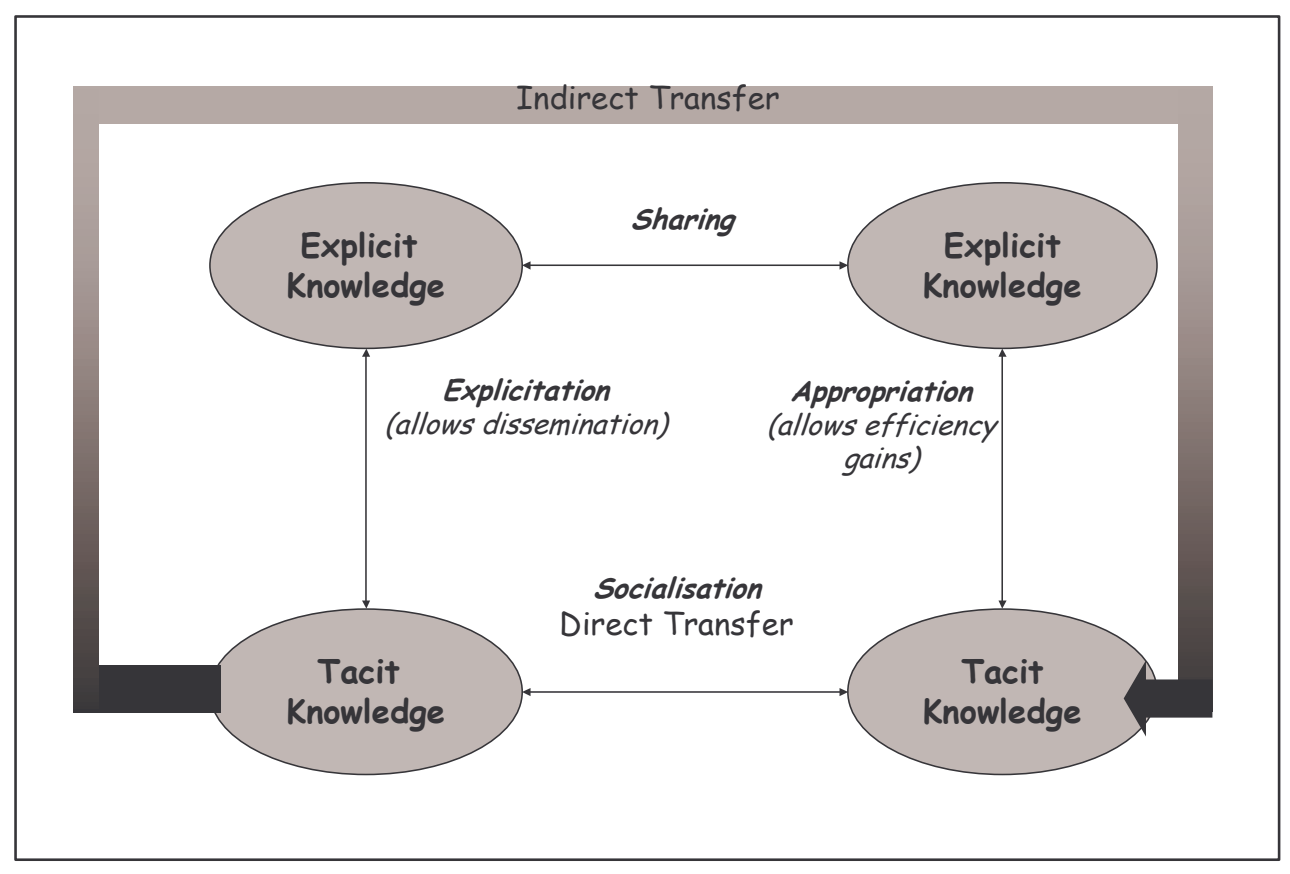

Figure 2: The Knowledge Capitalisation and Transfer Process

The direct transfer (socialisation) is a Knowledge Sharing Process without elicitation. An indirect transfer is an alternative way for Knowledge Sharing. The first sub-process is Knowledge Elicitation (or Elicitation or Acquisition). In the next sub-process, the Knowledge is shared. It is clear that Knowledge Elicitation is worthwhile only if the involved knowledge is shared among the concerned people within the organisation. The new information technologies (especially intranet) provide powerful tools for sharing knowledge, but it is not a sufficient condition: knowledge sharing is not obvious (Knowledge is power!), and leading change must be specifically designed for that purpose. The third sub-process is Knowledge Appropriation, where shared knowledge is used in operational way, in the working context, to perform complex tasks and/or solve problems. Experimentation, training are classical levers for that sub-process.

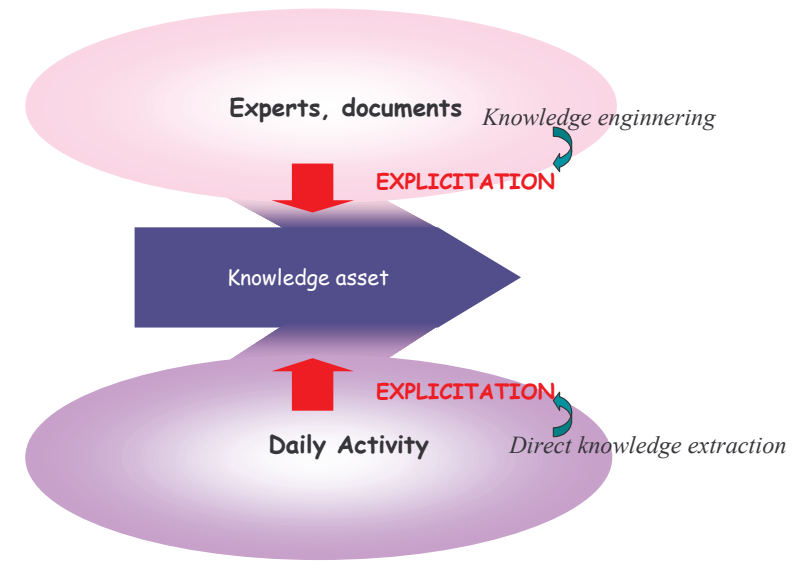

Figure 3: Two techniques to make knowledge explicit

There are two types of techniques that help to make knowledge explicit (Figure 3):

6) Direct knowledge extraction, in which knowledge is extracted directly and dynamically from organization activity. Data Mining, Text mining, traceability are some of these techniques. 
7) Knowledge capitalization, with which knowledge can be extracted by interviewing experts and from documents and modelled. Knowledge engineering methods are mainly used in this aim.

In this paper, knowledge capitalization approach are studied, especially the MASK Method (Method for Analysing and Structuring Knowledge). This method is used in many companies in France since 1996 [Ermine, 2000]. MASK delivers a final product called a "Knowledge Book".

\section{Knowledge capitalization success keys}

There is a number of knowledge capitalization methods [Dieng et al, 2000] inherited from knowledge engineering. These methods recommend knowledge modeling and provide methodologies (interviews, document analysis, modeling formalisms, etc.) to do that. Knowledge engineering approaches have been defined in the past, to develop knowledge based systems. For that, they recommend a systematic analysis in order to capture a model close as more as possible to the reality. The methodologies they recommend have been heavy in cost and time.

The objectives of knowledge capitalization are different. It aims at building organizational memories that represents several views on an expertise or activity. Knowledge must be capitalized in an easy way. So, a number of success keys corresponding on different phases of knowledge capitalization can be defined [Matta et al, 2001]:

- Knowledge extraction and formalization: Knowledge extraction and formalization must be done interactively with experts. Knowledge engineering and experts have to build together models. As consequence of this co-building: 1- knowledge engineering do not spend a lot of time in interviewing experts, analyzing and modeling ; 2- modeling formalism will be adapted and experts recognize easier models built and knowledge represented ; 3- experts consider models as a mirror of their knowledge that help them to abstract and formalize their reasoning in order to enrich models.

- Knowledge sharing: The big difference between a knowledge based system and an organization memory is that knowledge represented in this last one, must be shared by other organization actors. The main objectives of organization memory building are to keep track of problem solving experience and share it with others. So, the presentation form of knowledge memorized must take into account the internal representation of information and data in an organization. The knowledge engineering vocabulary as task, method, concept, etc. must be replaced by the specific activity formalism. In another hand, knowledge represented in models have to be shared and recognized unless by the experts teams. Meetings must be organized in order to discuss about a consensual knowledge representation.

- Knowledge reuse and appropriation: As noted above, knowledge is memorized in order to help in problem solving. So, the memory must offer an easy access to knowledge. For that, ergonomic procedures (several views, adapted and flexible presentations, etc.) must be used in order to facilitate this access. Note also, that communication technology can be used to integrate memory (or part of it) in actor workspace. The acceptability of the memory is an essential key of reuse. So, it is important to proceed by a number of training about its content and evaluate its reuse.

- Memory evolution: As a natural memory, an organization memory will evolve and knowledge capitalization process will be done continuously. Note also, that the structure of the memory will change as same as its content. So, memory must be built in a flexible way expecting changes and evolution.

The next section presents how the MASK method can satisfy these success keys. 


\section{MASK a method for knowledge capitalization}

MASK (Method of Analysis and Structuring Knowledge) is an evolution of the MKSM method. So, it is based on the same principles as MKSM [Ermine, 1996], [Barthelmé, 1998] and [Ermine, 2000]. The MASK method offers a flexible environment that allows to success knowledge capitalization projects. It has been applied in a large number of domains (safety, business process, mechanical design,) that have allowed its evolution.

\subsection{Knowledge extraction and formalization with Mask}

Model representation in Mask is inherited from knowledge engineering studies. So, it reflects a cognitive analysis of reasoning. Thus, it can be easy understood by an expert who recognizes a representation of its knowledge with models. Knowledge extraction and formalization can be so, done interactively with the expert. In fact, MASK recommends model co-building. Knowledge engineering presents a model and invites the expert(s) to fill it in. Expert(s) can suggest modifying some elements of the model and so on. For instance, when applying MASK to capitalize knowledge about woodworking machines safety, models have been titled by their content and not as activity model, task model and domain model. The expertise memorized has been presented as analysis of an incident, recommendation, danger phenomena, etc. Some elements also have been omitted in models as for example, the flux from danger phenomena models, etc. Knowledge capitalizing as recommended in MASK (with different point of views) guides expert to focus on a part of his world in order to describe it by emphasizing its main characteristics. Otherwise, it also helps him to have a global view of his expertise and problem solving experience. So, models can be considered as a mirror that push expert to enrich and complete models.

In another hand, there is no successive order of model building recommended in MASK. Each application domain has its formalization method. For example, in the woodworking machines safety application, knowledge engineering and expert start knowledge formalization by listing danger phenomena and modeling them. In another application which concerns business process services, activity models have been defined at first.

\subsection{Modelling tools}

Modeling tools as defined in MASK are generic enough and respond to expert's workspace and cognitive vision. These tools have an important role in knowledge extraction and formalization. They help to structure knowledge under: systemic, ergo-cognitive, psycho-cognitive, and historical and evolution analysis. Examples presented in the following are extracted from an experience of knowledge management in Woodworking machines safety. This experience has been done in the National Institute of Safety Research (INRS).

\subsubsection{The systemic analysis}




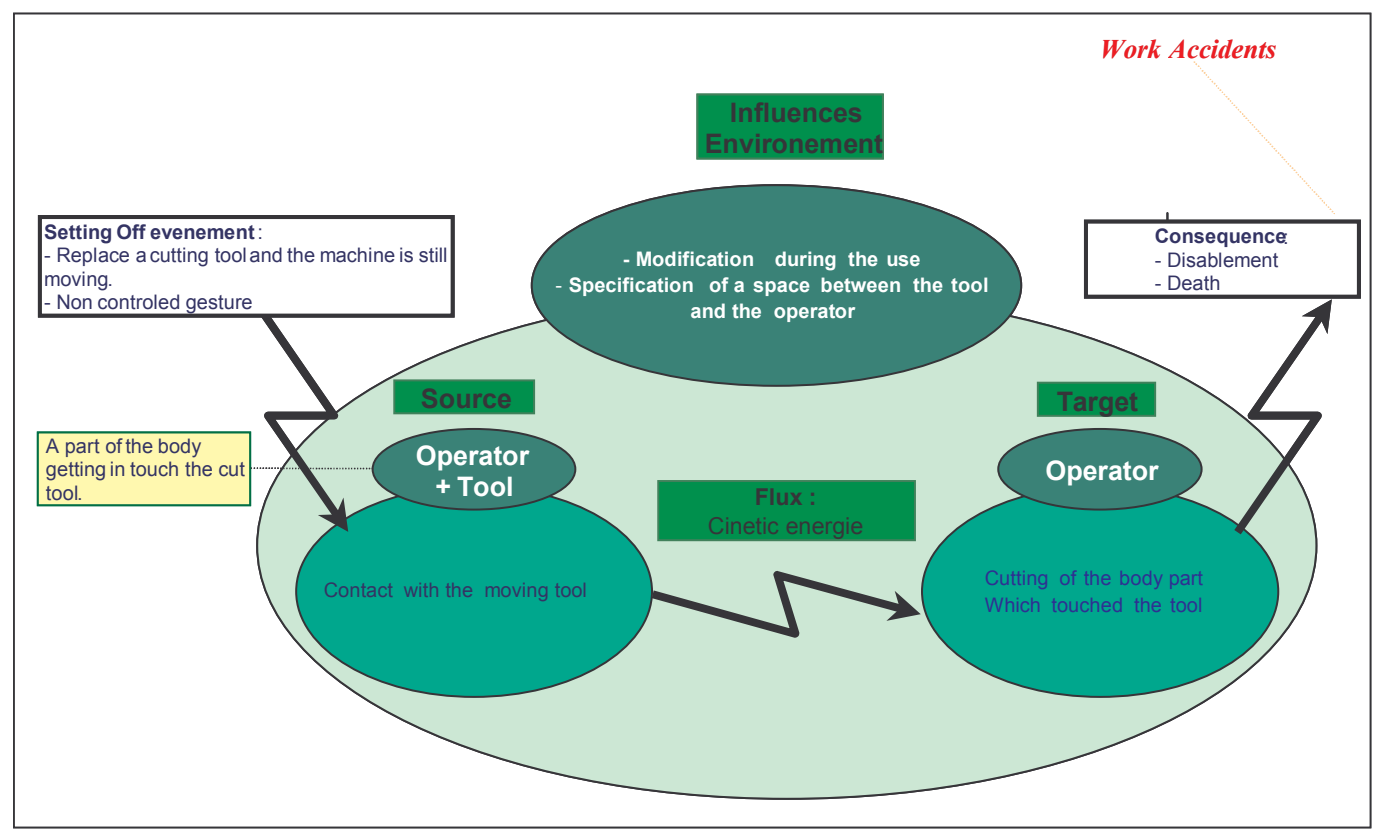

Figure 4: MASK a woodworking machines danger Phenomena model

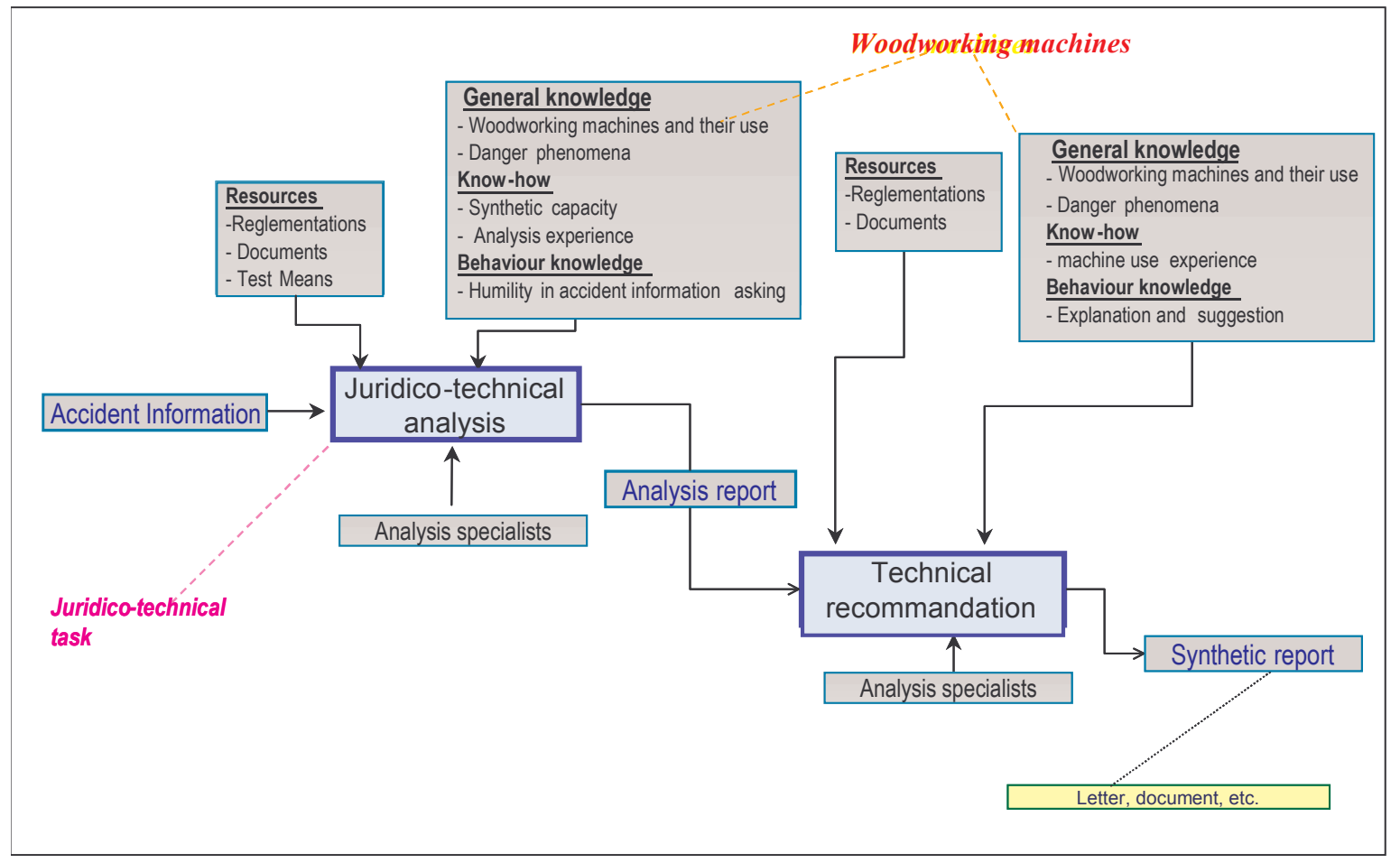

Figure 5: MASK: an incident analysis for woodworking machines safety

The hypothesis on which the question "What is the knowledge about?" relies upon is that the domain is described by a set of processes that take place into it (the system is "covered by processes"). Then, we have to produce a definition and description model of the different processes. The general system theory [Le Moigne, 1977], guiding MASK, sets two fundamental dualities. The first one is the flow/field duality. Every process produces its own flow that characterises it whatever the flow type is: material, energy, information or more generally cognitive, prescriptive or whatsoever. The notion of flow has to be kept together with the notion of field as it is in physics, where the field is seen as a "capacity of influence". That is, it represents all the elements that influence the process but do not belong to it (e.g. a catalyst in a chemical reaction, the 
wind and rain onto a fire). To sum up, we can say the flow takes place in the active process when the field takes place in the active environment

The second duality in the MASK process modelling is the source/target duality. The flow, in a process, links two subsystems. We call them: source and target. They both characterise the process as the flow and the field already did.

The processes may be linked together to form a scenario. Processes can be enhanced with an analytical form written by an expert where he puts all scientific elements (mathematical equations, range of data, threshold, chemical reactions...) that may help understanding the process. The scenarios and their set of processes with their analytical forms make the MASK phenomena model complete (Figure 4.).

The third stage of MASK is a system activity analysis. It takes into account the system that produces or consumes the knowledge. This model tries to link the domain knowledge with the operational situation of the system. This analysis is a top-down functional analysis where each activity can be hierarchically decomposed into some lower levels sub-activities. It is a data driven analysis; in a sense it is a simple structured description of the activities (of the system "functions") linked together by flows (usually data flows) they exchange. The language is close to the wildly spread SADT [IGL, 1982]. Then we will not detail it here. An example is given in (Figure 5).

\subsubsection{The ergo-cognitive analysis}

The task model (Figure 6) describes the "dynamic" knowledge and shows an ergo-cognitive analysis of an expertise. It is a representation of the strategy used to solve the problems due to the knowledge system. The questions that analysis wants to answer are: "Which kind of task should we solve?", "How this kind of task should usually be solved", "How should we use this static knowledge to solve our problem? » The MASK language used to build the task model gives the description of the tasks scheduling using a hierarchical recursive decomposition of a high level task into lower levels sub-tasks. Each non terminal task has a type which describes the way it controls its sub-tasks. A sequential task will execute its sub-tasks in the specified order. A parallel task will execute its sub-tasks at the same time. A conditional task will execute only its sub-tasks which condition is true. An iterative task will execute its sub-tasks in a loop changing each time their parameters. The tree that this model produces is called the control flow. This control flow is characteristic of the problem solving strategy and can be graphically presented (Figure 6).

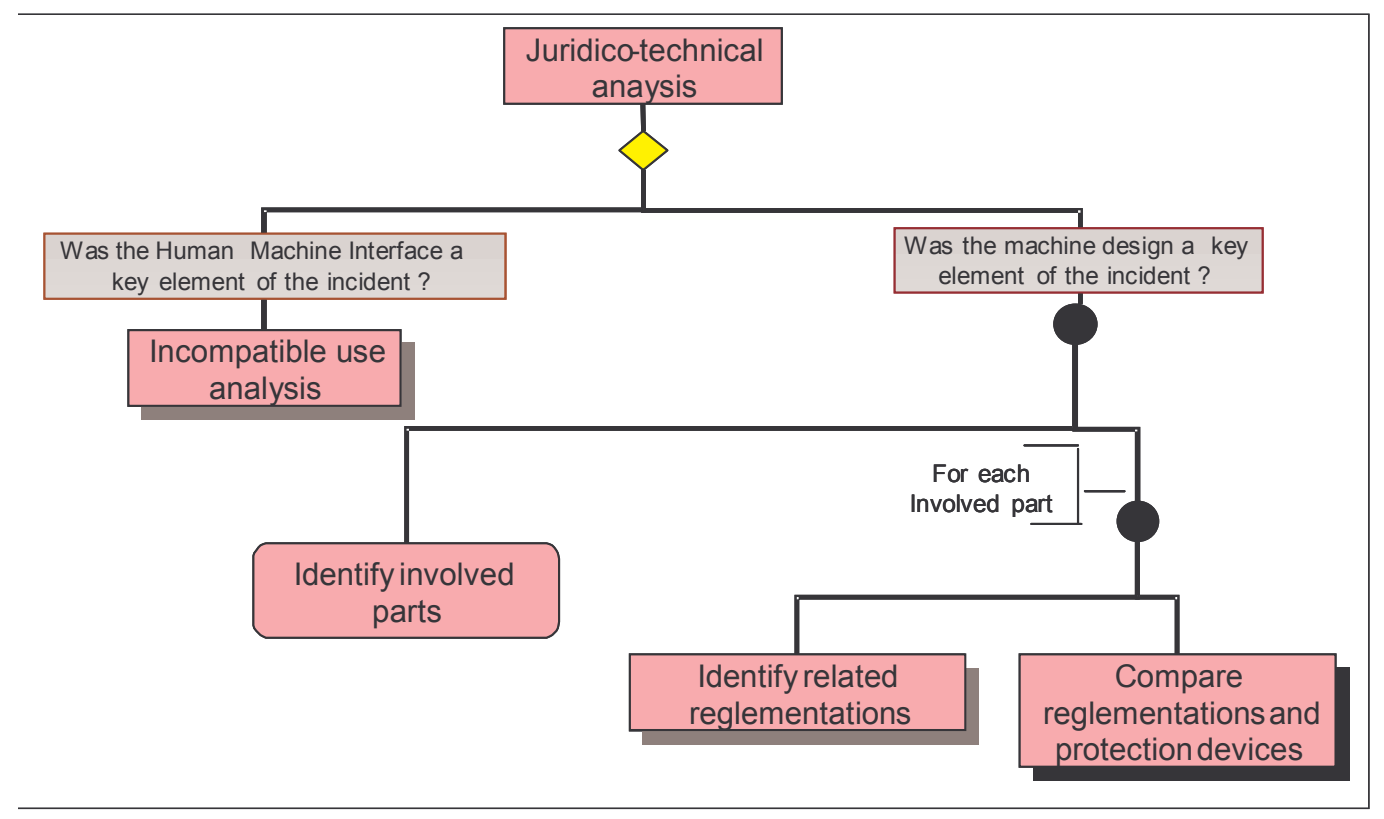

Figure 6: MASK: the Juridico-technical analysis modelling 


\subsubsection{The psycho-cognitive analysis}

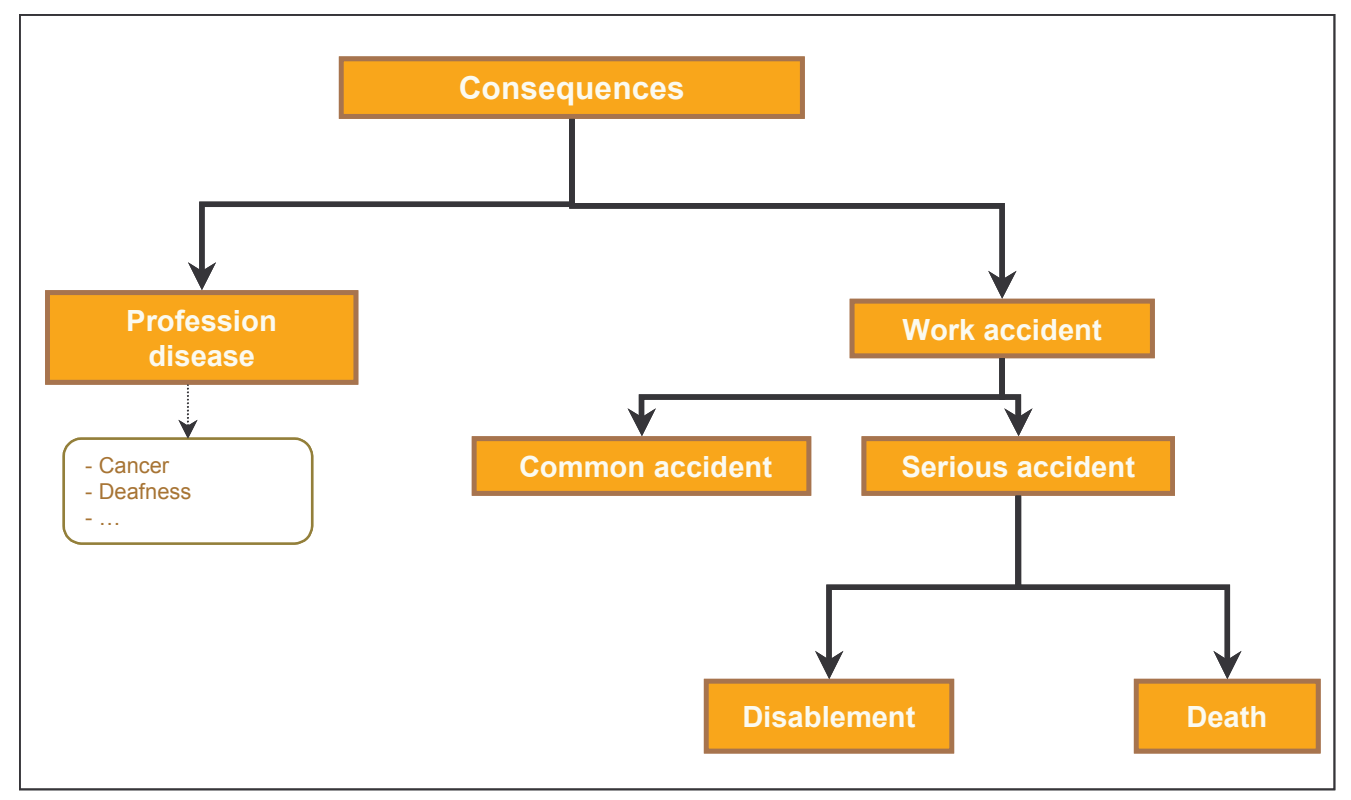

Figure 7: MASK: An accident consequences in woodworking machines use

The concept model (Figure 7.) gives the "static" aspect of the knowledge. To treat the problem, mainly two semantic mental structures exist that we call the network models and the properties models [Caron, 1989]. In knowledge engineering vocabulary, we call them respectively semantic network and object-oriented diagram. When the object-oriented diagram enhances the properties embedding them with the concept they describe, the semantic network enhances the relationships between the concepts. These two models can often be considered as equivalent, under certain circumstances. The concepts model mixes the two points of view.

An object depicts a real world entity. As this entity is unique, the object is unique as well, and hence, has its own identity. A concept depicts a category of objects which all have the same properties. An object belongs to a concept; we then say it is an instance of this concept. The common properties of a concept are represented by its attributes. Then we want to describe how the concepts are hierarchically organised. For this purpose we use the specialisation relationship. Finally we have a value relationship that gives the possible values a concept can have

\subsubsection{The evolution analysis}

Knowledge System evolution can be partly modelled by the context defined by history of mutations and accommodations. The historical analysis aims at describing the evolution of objects and techniques among years and experiences. So, it provides a contextual representation of the evolution.

In this type of analysis, technologies and objects will be classified in order to represent their evolution among milestones. Objectives underlining each milestone can be also identified. The influence between elements is emphasizing as arrows (Figure 8).

Another model (called generation model) is defined in order to emphasis concept bases of a knowledge system. It reflects a mental analysis of concept evolution (how and why). This model is adapted from technology history theory [Deforge, 1985]. The generation model describes in fact, a concept or object succession that corresponds to a progression of techniques and their amelioration. This type of model (Figure 9) represents the evolution of a generation: mutation, alteration, bifurcation, interruption, etc. Main evolution motive is also showed in this model. There are also a number of argumentation elements which describes even positive and negative consequences of each generation. 


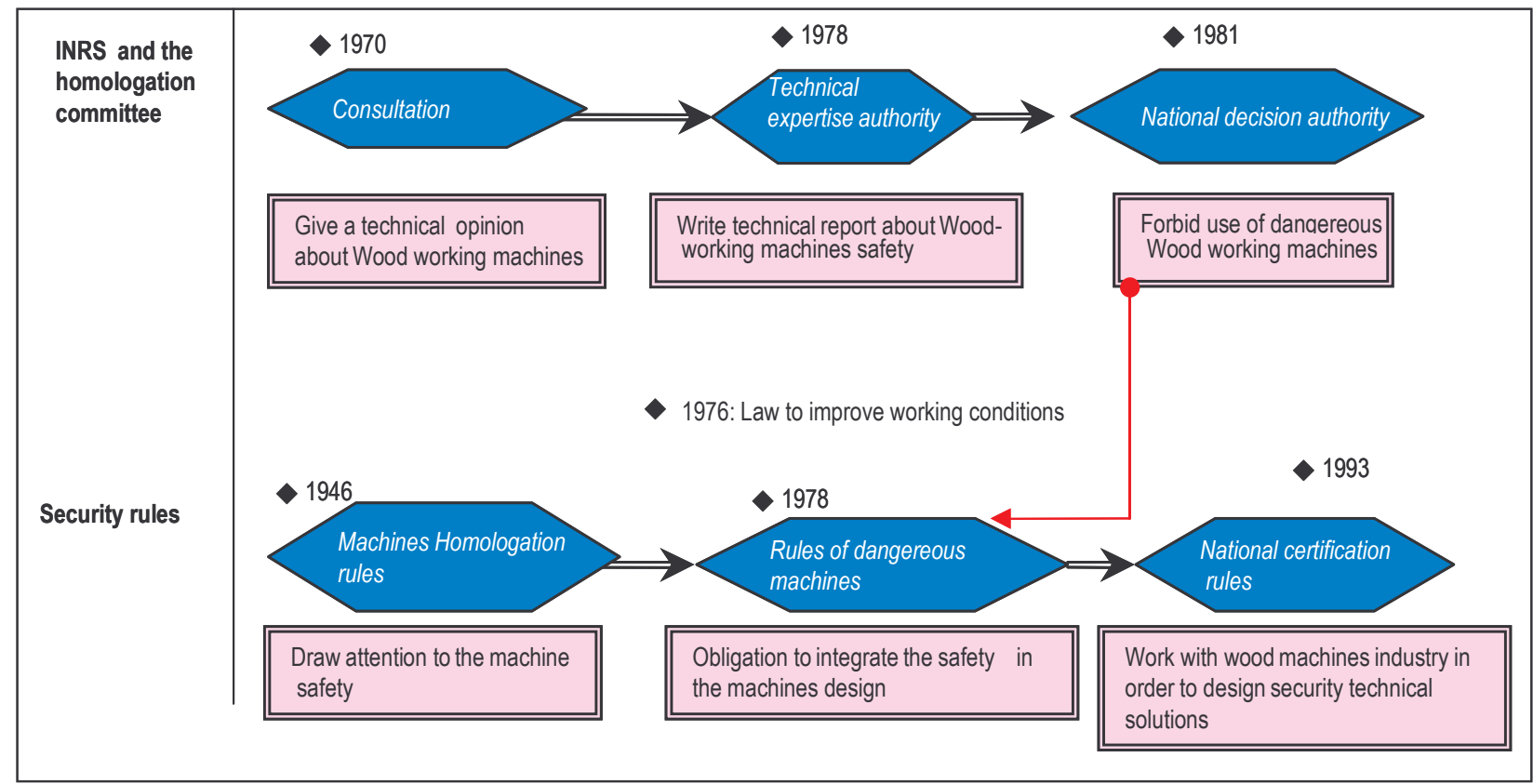

Figure 8: The historic track of safety rules and homologation in woodworking machines analysis.

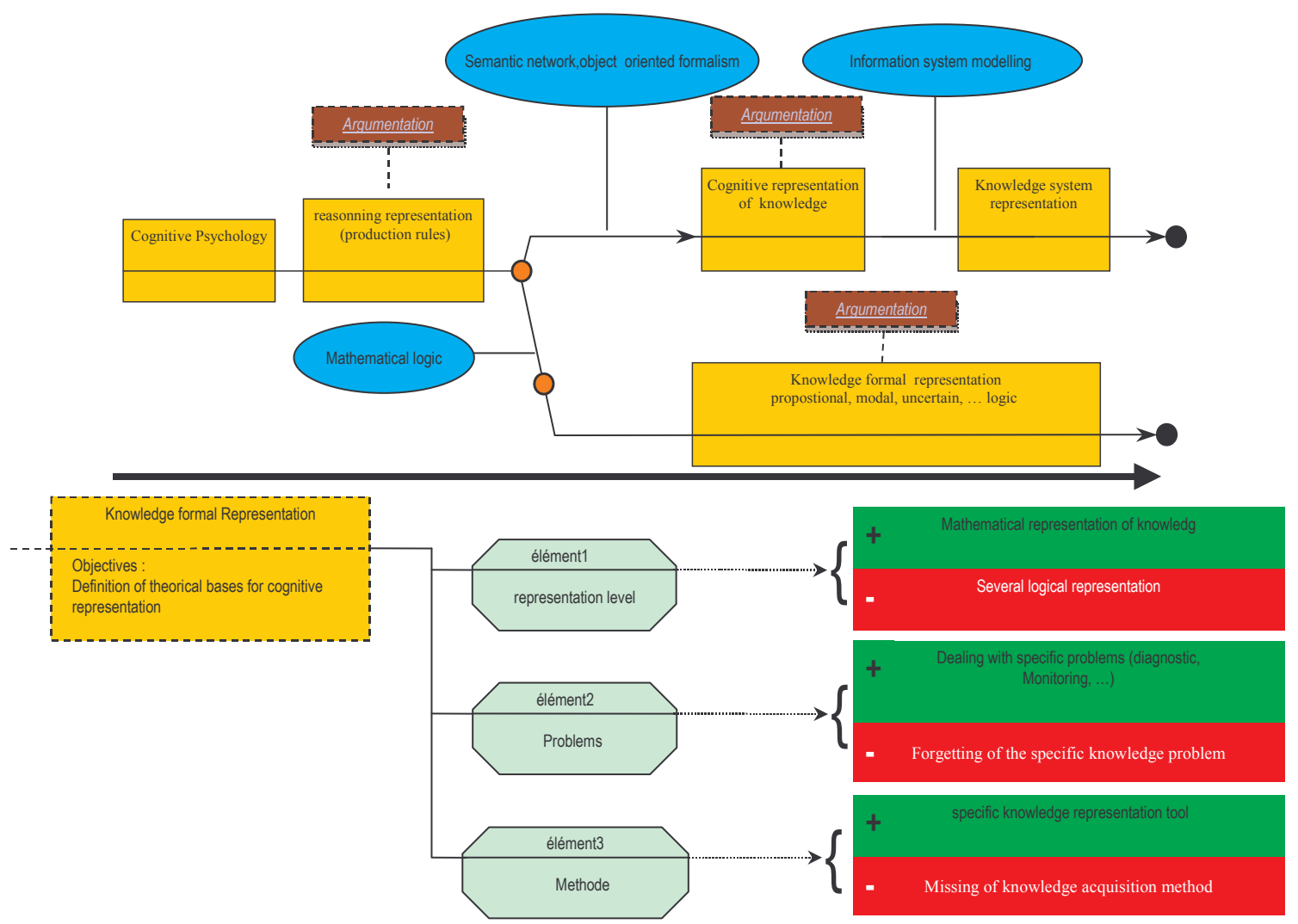

Figure 9: An example of Generation model with MASK (adapted from knowledge engineering application) 


\subsection{Knowledge sharing with MASK}

The memory, built by MASK, is considered as a knowledge book which can be edited as a real book. So, it is necessary to follow a specific procedure: submission, reviewing content and presentation and publishing under different aspects, respecting users' needs, workspace and habits.

For instance, the organization memory built for the woodworking machine safety, is presented under two aspects: a paper book (Figure 10) and multimedia knowledge book. In machines safety analysis, paper books are usually considered as good references. Analysts use also Hypermedia tools (especially the Web). So, we defined also a multimedia knowledge book which gathers models, definitions and references with hypermedia access facilities.

Knowledge sharing does not consist only to present knowledge to users, but they have to recognize it as a reference. MASK recommends organizing consensual meetings in order to invite actors, especially experts' teams to recognize knowledge kept as reference of some of their activities. With these meetings a knowledge network can be defined. This network help actors to know which part of the memory can be reused in their activity and when. Meetings will be also organized with hierarchical managers that can approve knowledge book as references of the organization.

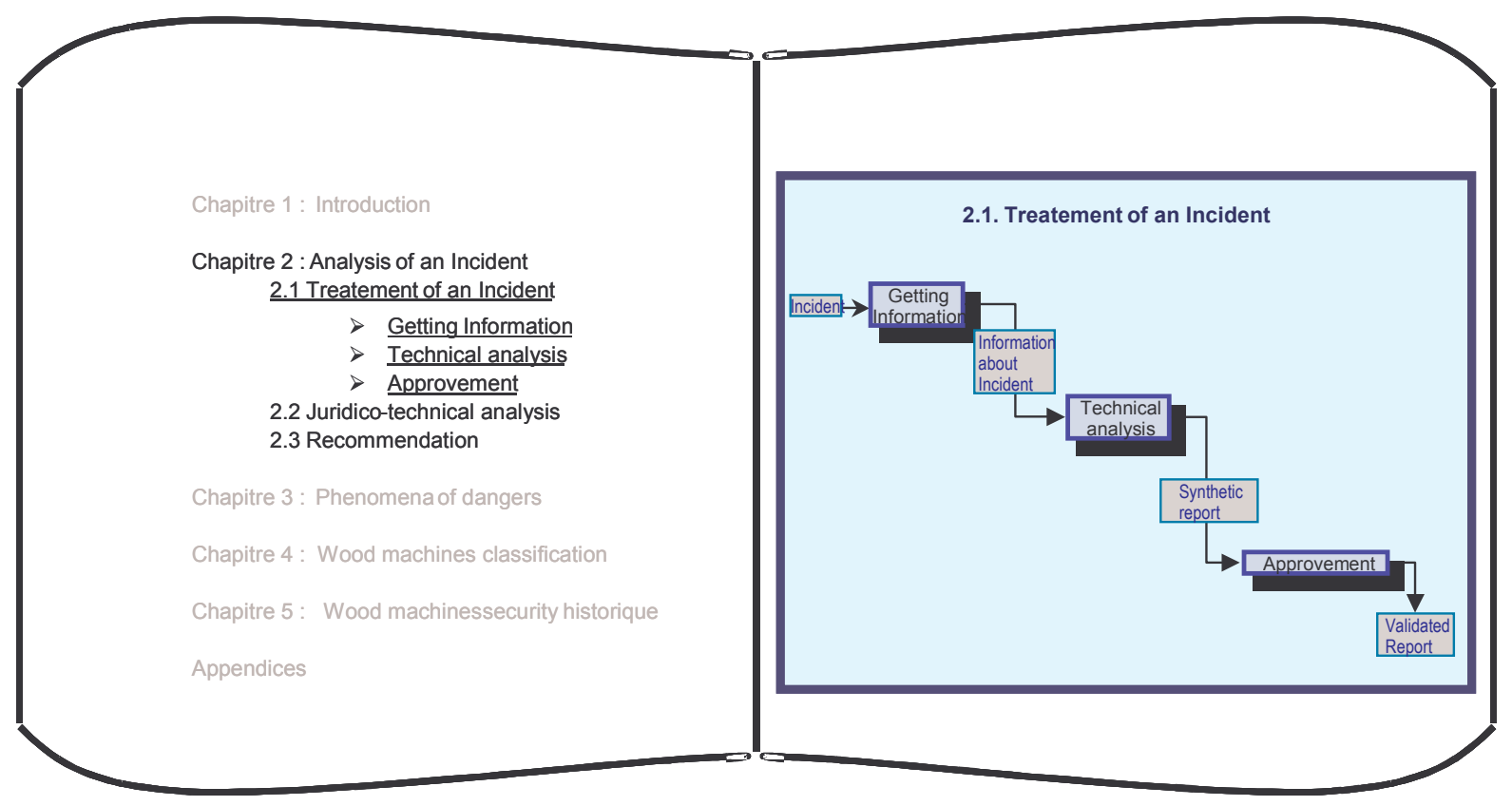

Figure 10: Paper knowledge book presentation

\section{Towards a knowledge reuse and appropriation}

As a first step to facilitate memory reuse, we recommend some ergonomic procedures to specify knowledge access. These procedures will be specific for each form of the knowledge book: paper, hypermedia, etc. We studied only some navigation principles in this aim. We propose models as a base of this navigation. As noted above, these models were inherited from cognitive analysis, so, that they can be accessible by a large number of users. For instance, this type of navigation has been privileged in the paper book defined in woodworking machines safety memory (Figure 10) and also in business process services one.

Hypermedia techniques provide several ways of access. In the woodworking machines safety memory, we defined in addition of the navigation by models (cognitive schema), navigation by theme (structural schema) (Figure 11). Other types of navigation can be also studied corresponding to users' workspace and needs. 
The model presentation in MASK is also based on a color chart which helps to distinguish the duality of knowledge. Knowledge, with MASK, is characterized respecting two dualities: context versus signification, functions versus structure. So we use the duality of colors to present indirectly the knowledge type duality (Figure 11). In fact, activity and phenomena describe the context of an expertise; task and concept represent the semantic. Otherwise, activity and task are functional description and phenomena and concept are structured one.

In addition, techniques to organize training sessions and to promulgate knowledge reuse in order to facilitate knowledge sharing can be proposed. Memory reuse must be evaluated and knowledge representation must be modified consequently. We have not yet studied memory reuse evaluation and knowledge appropriation. Ergonomic activity analysis will give some guidelines to study knowledge appropriation process.

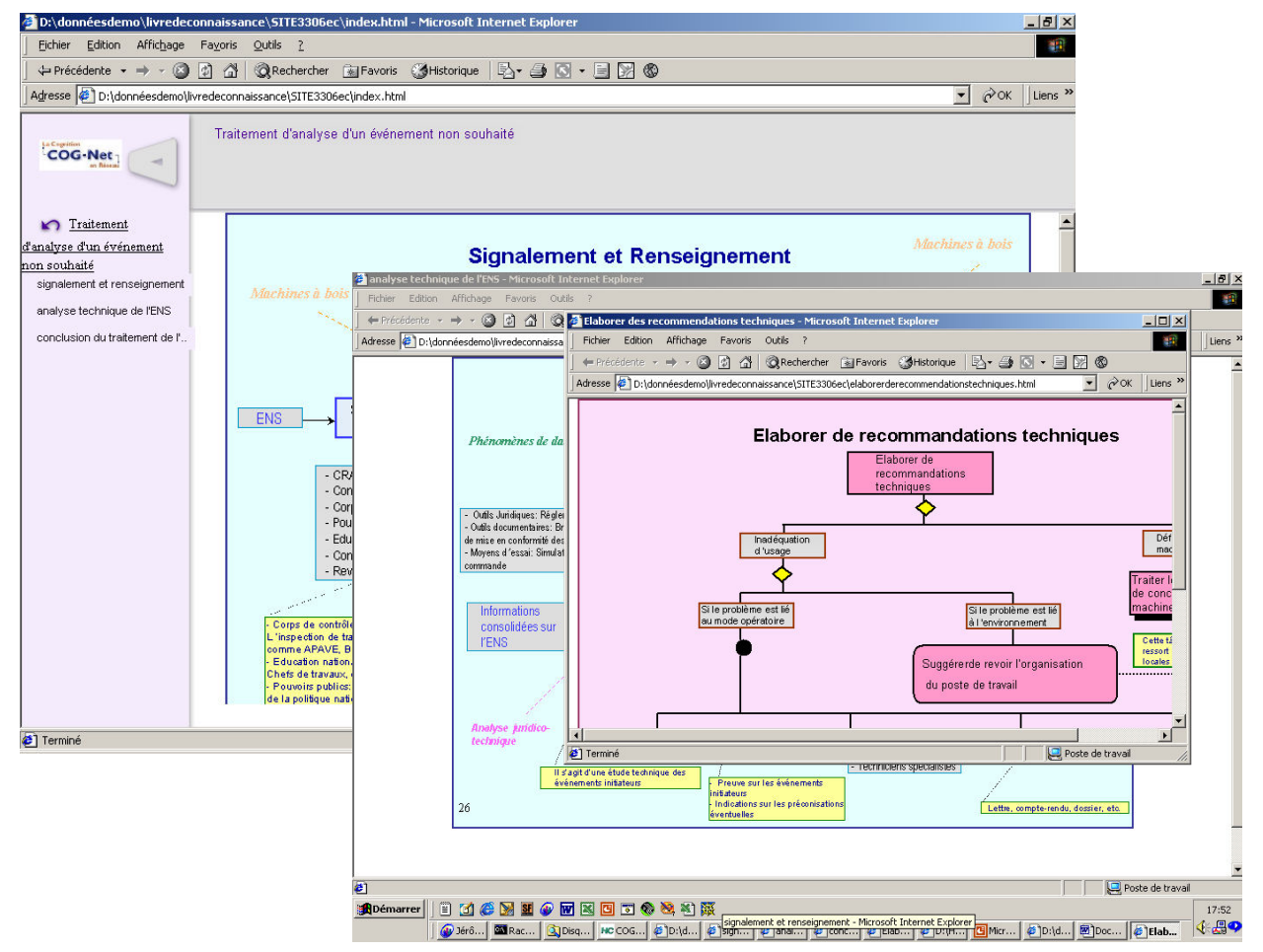

Figure 11: Hypermedia knowledge book

\section{Conclusion}

Knowledge is considered nowadays as the main asset of an enterprise. The know-how of actors in an organization is an important concurrence factor. There are a number of approaches that studied how this knowledge can be capitalized in an organization. Some of these approaches have inherited techniques from knowledge engineering, especially methodological and modeling guidelines. But these guidelines cannot be used as directly suggested in knowledge engineering techniques. The objectives of knowledge capitalization are different from building knowledge based systems in which the knowledge must be coherent, operational and treat a large number of problems. 


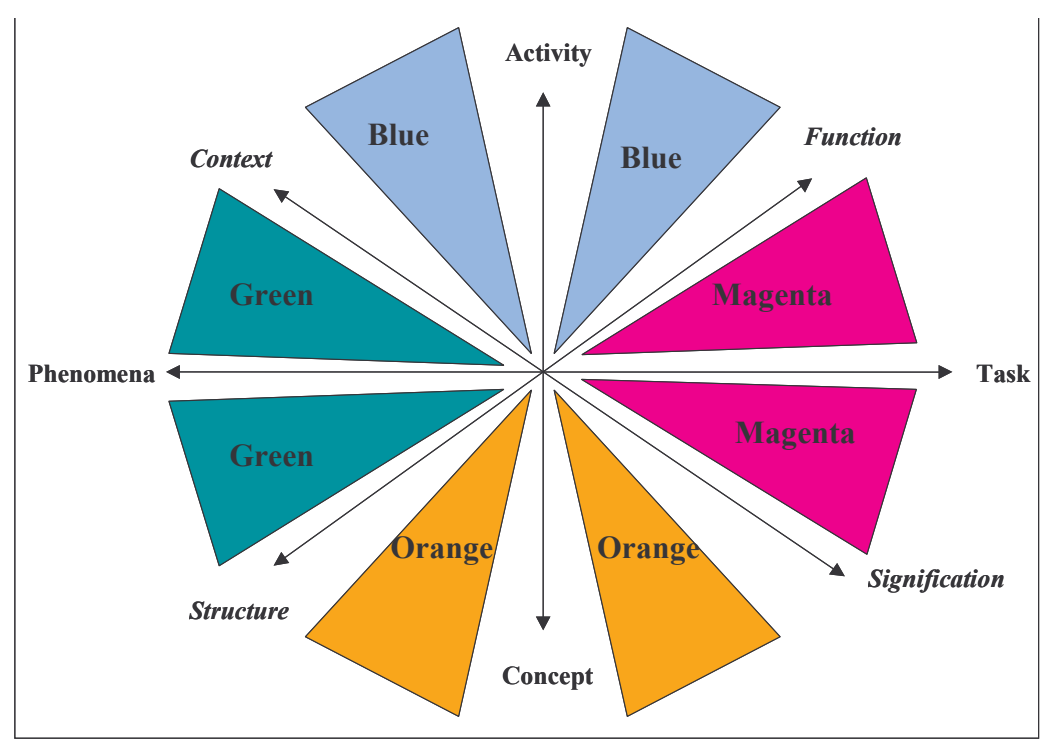

Model presentation based on colour dualities

Knowledge capitalization methods must offer a light process that can be used without perturbing the organization activity. We defined in this paper a number of success keys that can help a knowledge engineer to capitalize knowledge in this way. We can summarize these keys by:

- Co-modeling done interactively by knowledge engineer and expert(s),

- Mirroring phenomena: expert(s) recognize models at a mirror of his knowledge,

- Homogeneous presentation of models corresponding with actors languages and formalism,

- Memory so built must be unless recognized by expert(s) teams and approved by the hierarchy,

- Use of ergonomic procedures and communication means in order to facilitate memory access and sharing,

- Memory must be built in a flexible way expecting changes and evolution.

The MASK method has been defined in order to respect these keys. Its application in several domains has proved its success and its respect of some of the keys noted above. Especially, the co-modelling tackled by model guided interviews, the mirroring phenomena enhanced by the generic structure of models that corresponds to the structural and cognitive representation of a human activity, the homogeneous presentation with actor languages due to the flexibility of the model representations and modelling tools, the tackling of knowledge sharing enhanced by a presentation under different points of view and use of several memory supports (paper, hypermedia, etc.).

We are studying techniques to enhance knowledge reuse and to evaluate its contribution in an organization. Our analysis of the evolution theory will guide us to study the evolution of an organizational memory and to support it by a dynamic method. We also deal with the influence of the knowledge history in the innovation.

\section{1. $5 . \quad$ REFERENCES}

[Barthelmé et al,1998] Barthelmé F., Ermine J.L., Camille Rosenthal-Sabroux An architecture for knowledge evolution in organisations, European Journal of Operational Research 109, 414-427 (1998)

[Caron, 1989] Caron J.: Précis de psycholinguistique, P.U.F, Paris, 1989

[Dieng et al, 2000] Dieng R., Corby O., Giboin A., Golebiwska J., Matta N., Ribière M., Méthodes et outils pour la gestion des connaissances, Dunod., 2000

[Deforge, 19 85] Y. Deforge : Technologie et génétique de l'objet industriel, Maloine éd., Paris, 1985 
[Ermine, 1996] J-L. Ermine, M. Chaillot, P. Bigeon, B. Charreton, D. Malavieille : MKSM, a method for knowledge management, Knowledge Management, Organization, Competence and Methodology, Advances in Knowledge Management Volume 1, Jos. F. Shreinemakers Ed., pp 288 - 302, Ergon, 1996

[Ermine, 2000] Ermine J-L: Les systèmes de connaissances, Edition Hermès, Paris, 2000

[IGL, 1982] IGL, France : Introduction à SADT, Manuel, 1982

[Le Moigne, 1977] Le Moigne J-L : La théorie du système général, P.U.F.,1977,2 ${ }^{\text {nd }}$ edition. 1984

[Matta et al, 2001] Matta N. Ermine J.L., Aubertin G., Trivin J.Y., Knowledge Capitalization with a knowledge engineering approach : the MASK method, proceedings of IJCAI'2001 Workshop on Knowledge Management and Organizational Memory, August 2001.

[Nonaka, 1995] I. Nonaka, H. Takeuchi: The knowledge-Creating Company: How Japanese Companies Create the Dynamics of Innovation. Oxford University Press, 1995 